\title{
Analysis of Gait Motion Changes by Intervention Using Robot Suit Hybrid Assistive Limb (HAL) in Myelopathy Patients After Decompression Surgery for Ossification of Posterior Longitudinal Ligament
}

\author{
Seioh Ezaki ${ }^{1 *}$, Hideki Kadone ${ }^{2}$, Shigeki Kubota ${ }^{1}$, Tetsuya Abe ${ }^{1}$, Yukiyo Shimizu ${ }^{2,3}$, \\ Chun Kwang Tan ${ }^{4}$, Kousei Miura' ${ }^{1}$, Yasushi Hada ${ }^{3}$, Yoshiyuki Sankai ${ }^{2,4}$, Masao Koda ${ }^{1,2}$, \\ Kenji Suzuki ${ }^{2,4}$ and Masashi Yamazaki ${ }^{1}$ \\ ${ }^{1}$ Department of Orthopaedic Surgery, University of Tsukuba, Tsukuba, Japan, ${ }^{2}$ Center for Cybernics Research, University of \\ Tsukuba, Tsukuba, Japan, ${ }^{3}$ Department of Rehabilitation Medicine, University of Tsukuba, Tsukuba, Japan, ${ }^{4}$ Faculty of \\ Engineering, Information and Systems, University of Tsukuba, Tsukuba, Japan
}

OPEN ACCESS

Edited by:

Andrés úbeda,

University of Alicante, Spain

Reviewed by:

Guillaume Durandau,

University of Twente, Netherlands

Zha Fusheng,

Harbin Institute of Technology, China

*Correspondence:

Seioh Ezaki

s1937003@s.tsukuba.ac.jp

Received: 06 January 2021 Accepted: 22 February 2021

Published: 31 March 2021

Citation:

Ezaki S, Kadone H, Kubota S, Abe T,

Shimizu Y, Tan CK, Miura K, Hada Y,

Sankai Y, Koda M, Suzuki K and

Yamazaki M (2021) Analysis of Gait

Motion Changes by Intervention Using

Robot Suit Hybrid Assistive Limb

(HAL) in Myelopathy Patients After

Decompression Surgery for

Ossification of Posterior Longitudinal

Ligament.

Front. Neurorobot. 15:650118.

doi: 10.3389/fnbot.2021.650118
Ossification of the posterior longitudinal ligament (OPLL) is a hyperostonic condition in which the posterior longitudinal ligament becomes thick and loses its flexibility, resulting in ectopic ossification and severe neurologic deficit (Matsunaga and Sakou, 2012). It commonly presents with myelopathy and radiculopathy and with myelopathy progression motor disorders and balance disorders can appear. Even after appropriate surgical decompression, some motor impairments often remain. The Hybrid Assistive Limb (HAL) is a wearable powered suit designed to assist and support the user's voluntary control of hip and knee joint motion by detecting bioelectric signals from the skin surface and force/pressure sensors in the shoes during movement. In the current study, the HAL intervention was applied to 15 patients diagnosed with OPLL who presented with myelopathy after decompression surgery (6 acute and 9 chronic stage). Following the HAL intervention, there were significant improvements in gait speed, cadence, stride length, in both acute and chronic groups. Joint angle analysis of the lower limbs showed that range of motion (ROM) of hip and knee joints in acute group, and also ROM of hip joint and toe-lift during swing in chronic group increased significantly. ROM of knee joint became closer to healthy gait in both groups. Electromyography analysis showed that hamstrings activity in the late swing phase increased significantly for acute patients. Immidiate effect from HAL session was also observed. EMG of vastus medialis were decreased except chronic 7th session and EMG of gastrocnemius were decreased except acute 7th session, which suggests the patients were learning to walk with lesser knee-hypertension during the sessions. After all, double knee action appeared in both acute and chronic groups after the HAL intervention, rather than knee hyper-extension which is a common gait impairment in OPLL. We consider that these improvements lead to a smoother and healthier gait motion.

Keywords: hybrid assistive limb, ossification of the posterior longitudinal ligament, rehabilitation, postoperative procedures, robotics, gait analysis 


\section{INTRODUCTION}

Myelopathy refers to a pathology that causes neurological deficits in the spinal cord (Oyinkan Marquis and Capone, 2016), that usually results from compression of the spinal cord by osteophytes or extruded intervertebral disks between the cervical spine (Seidenwurm, 2008). Age-related degeneration of the tissues in the spinal column, known as degenerative myelopathy, is the most common cause of spinal cord dysfunction among older adults. The structural changes include: degeneration of intervertebral disc, vertebral bodies, and facet joints; hypertrophy of the ligament flavum; and ossification of the posterior longitudinal ligament (OPLL) (Fehlings et al., 2017). OPLL is a hyperostonic condition characterized by pathological ectopic ligament ossification that mostly affects the cervical or thoracic spine segments (Epstein, 2002; Matsunaga and Sakou, 2012) and commonly presents with myelopathy and radiculopathy. OPLL was previously considered specific to Asian population (Mizuno and Nakagawa, 2006), and the Japanese have a significantly high incidence rate of this disease (Matsunaga and Sakou, 2006); however, its prevalence among Caucasians has been increasing recently (Fehlings et al., 2015). Motor disorders and balance disorders can appear with myelopathy progression. Spinal stenosis syndrome, which largely stems from motor weakness, can occur during or after middle age (McKinley et al., 1998). When symptoms are mild, observation, physical therapy, and oral analgesics are chosen as a means of non-operative management. However, when myelopathy progresses or is irresponsive to conservative management, surgical management is usually adopted. Surgery is thought to be an effective treatment for patients with myelopathy or severe stenosis (Epstein, 2014). However, even after appropriate decompression operation has been successfully achieved, patients are sometimes left with residual motor impairments. The long-term postoperative prognosis tends to be a gradual reworsening of neurological symptoms after 5 years (Committee for Clinical Guidelines of the Japanese Orthopaedic Association, 2011). Several methods for gait rehabilitation are available for patients with myelopathy following surgical decompression including muscle training, range of motion training, movement equilibrium training, and sphincter control. Recently, there has been a lot of discussion about the potentials and limitations of treadmill-based rehabilitation. Several studies have shown that there are no advantages to using passive robotic rehabilitation such as treadmill walking (Hidler et al., 2009) compared to conventional rehabilitation therapy (Dobkin et al., 2006).

Recent studies have reported the use of the exoskeleton robot suit, the Hybrid Assistive Limb (HAL) (Cyberdyne, Tsukuba, Japan) as a novel approach to manage gait rehabilitation (Sankai and Sakurai, 2018). HAL is a wearable powered suit, designed to assist and support users' voluntary control of hip and knee joint motion by detecting bio-electric signals (muscle action potentials) from surface electrodes and from force/pressure sensors in the shoes (Kawamoto and Sankai, 2005; Sankai and Sakurai, 2018). Compared to other robot training, HAL can enhance the wearer's voluntary motion, and intensify its feedback. Recent studies have reported the feasibility and effectiveness of HAL for functional recovery in rehabilitation use for multiple neurological disorders patients with gait disturbance after spinal cord diseases and cerebrovascular diseases such as chronic stroke (Kawamoto et al., 2013; Nilsson et al., 2014; Wall et al., 2015; Kasai and Takeda, 2016), chronic spinal cord injury (Kubota et al., 2013; Aach et al., 2014; Sczesny-Kaiser et al., 2015; Wall et al., 2015; Ikumi et al., 2017), and OPLL (Sakakima et al., 2013; Kubota et al., 2016, 2019; Fujii et al., 2017; Puentes et al., 2018). For the chronic stage of myelopathy in patients with postoperative cervical OPLL, improvement in gait speed, step length, and cadence has been observed (Kubota et al., 2016). Furthermore, it has enabled patients to use the knee extensor in the stance phase without locking the knee by supporting gluteus maximus and quadriceps muscle activation (Shimizu et al., 2017, 2018).

The purpose of rehabilitation robotics is not to replace human therapists, but to provide a tool to increase productivity (Tejima, 2001) and deliver appropriate rehabilitation to more patients. This technology increases the repeatability of movement and also provides tools to assess the progress and effectiveness of the rehabilitation processes (Huang and Krakauer, 2009).

Also, achieving a faster gait speed through rehabilitation should be done while ensuring that no compensational gait is developed (Verma et al., 2012). If unhealthy gait such as hyper knee extension develops, it is more likely to result in locomotive syndrome or the need for further surgical intervention (Bleyenheuft et al., 2010; Mao et al., 2015). As the population is aging and the life expectancy after surgery is lengthening, there is an increasing need for maintaining a good quality of life through the development of a healthier gait.

In this study, we analyse the joint angles and muscle activities during gait before and after HAL gait intervention, and report changes therein and also improvements of double knee action and walking ability, in 15 patients with postoperative OPLL (6 acute and 9 chronic stage) who presented myelopathic gait in their acute and the chronic stage after decompression surgery.

\section{METHODS}

\subsection{Participants}

Fifteen patients who had undergone decompression surgery after diagnosed with OPLL associated with severe motor impairment (Table 1). Six of them ( 3 male and 3 female) received the HAL intervention at an acute stage of postoperative gait disorder. The mean age of the patients at an acute stage was 57.8 years old, and HAL intervention was started at a mean of 25 days after surgery. The other nine patients $(9$ males) received the intervention at a chronic stage of postoperative gait disorder. The mean age of the patients at a chronic stage was 68.3 years old and HAL intervention was started at a mean of 1023.2 days after surgery. Kinematic data from eight healthy volunteers ( 3 male, 5 female) who did not receive HAL treatment was used for comparison as a healthy gait. The mean age of healthy volunteers was 57 years old.

Each of the 15 patients was provided with 10 HAL gait rehabilitation intervention sessions. Each HAL session lasted 
TABLE 1 | Subject characteristics.

\begin{tabular}{|c|c|c|c|c|c|c|}
\hline Participant ID & Group & Sex & Age & Height (cm) & Weight (kg) & Surgery-HAL interval (days) \\
\hline A1 & Acute & $\mathrm{F}$ & 78 & 146 & 51 & 15 \\
\hline $\mathrm{A} 2$ & Acute & $\mathrm{M}$ & 64 & 165 & 90 & 26 \\
\hline A3 & Acute & $\mathrm{M}$ & 52 & 180 & 100 & 18 \\
\hline A4 & Acute & $\mathrm{F}$ & 63 & 154 & 56 & 32 \\
\hline A5 & Acute & $\mathrm{F}$ & 41 & 156 & 79 & 31 \\
\hline A6 & Acute & $\mathrm{M}$ & 49 & 169 & 80 & 28 \\
\hline C1 & Chronic & M & 70 & 168 & 71 & 288 \\
\hline C2 & Chronic & M & 75 & 168 & 78 & 287 \\
\hline C3 & Chronic & $\mathrm{M}$ & 68 & 174 & 64 & 3655 \\
\hline C4 & Chronic & $\mathrm{M}$ & 78 & 159 & 61 & 372 \\
\hline C5 & Chronic & $\mathrm{M}$ & 76 & 166 & 63 & 2188 \\
\hline C6 & Chronic & $\mathrm{M}$ & 58 & 176 & 72 & 540 \\
\hline $\mathrm{C} 7$ & Chronic & $\mathrm{M}$ & 66 & 157 & 74 & 730 \\
\hline C8 & Chronic & $\mathrm{M}$ & 70 & 163 & 69 & 958 \\
\hline C9 & Chronic & $\mathrm{M}$ & 44 & 174 & 100 & 191 \\
\hline $\mathrm{H} 1$ & Healthy & $\mathrm{F}$ & 56 & 157 & 59 & - \\
\hline $\mathrm{H} 2$ & Healthy & $\mathrm{F}$ & 42 & 161 & 49 & - \\
\hline H3 & Healthy & $\mathrm{F}$ & 59 & 152 & 53 & - \\
\hline $\mathrm{H} 4$ & Healthy & $\mathrm{F}$ & 67 & 164 & 50 & - \\
\hline H5 & Healthy & $\mathrm{F}$ & 60 & 158 & 57 & - \\
\hline H6 & Healthy & $\mathrm{M}$ & 50 & 163 & 58 & - \\
\hline $\mathrm{H} 7$ & Healthy & $\mathrm{M}$ & 45 & 164 & 61 & - \\
\hline H8 & Healthy & $\mathrm{M}$ & 77 & 170 & 59 & - \\
\hline H9 & Healthy & $\mathrm{M}$ & 66 & 166 & 62 & - \\
\hline
\end{tabular}

Surgery-HAL interval refers to the number of days elapsed from the surgery to the beginning of HAL therapy.

approximately $1 \mathrm{~h}$, which included $20 \mathrm{~min}$ of HAL-assisted walking. The HAL assist parameters were configured to the patient's comfort for each session. The All-in-One walking device (Ropox Inc., Denmark) was used alongside the HAL. When necessary, weight support was provided for the acute patients so that they could walk by themselves. Signed informed consent was obtained from each participant after they received a full explanation about the present research and its data usage. This study was approved by the University of Tsukuba Hospital Ethics Committee (Approval number: H26-22).

\subsection{HAL Set up}

The double-legged version of the HAL suit was used for this study. This HAL suit has a total of four electric motors, located bilaterally at the patient's hip and knee joints, which assist the wearer's leg motion and gait. The hip and knee motors were actuated for producing torque in proportion to a weighted difference of the respective muscles. Activation of these motors is guided in real time by neuromuscular activities detected by surface electrodes placed at the iliopsoas (hip flexor), gluteus maximus (hip extensor), biceps femoris (knee flexor), and quadriceps (vastus lateralis and knee extensor).

\subsection{HAL Intervention}

The HAL intervention included 10 sessions of HAL therapy conducted twice per week at 90 min per session. Patients at an acute stage of disease progression began HAL therapy during their hospitalization period, while those at a chronic stage began as outpatients. Each session began with fitting the HAL suit. For those who needed, a walking device (All-in-One Walking Trainer, Ropox A/S, Naestved, Denmark) with a harness was provided to support body weight and prevent falling. The HAL therapy session consisted of 20 min of walking at a comfortable pace on a $25-\mathrm{m}$ oval-shaped walking course with rest intervals. Vital signs including blood pressure, heart rate, and oxygen saturation were recorded at the beginning, during, and end of each session to ensure that patients were stable. The patients' lower limb joint control ability was assessed by a clinician before and after the HAL intervention using MMT (Manual muscle testing) scale grading $0-5$.

\subsection{Gait Measurement \\ 2.4.1. Joint Angles}

The functional evaluation and the walk tests were carried out without the participant wearing the HAL suit before the first session and after the last one. The time and the number of steps were counted while they walked $10 \mathrm{~m}$ in a straight line at a comfortable pace, and the speed and stride length were calculated using this data. Gait before and after the HAL intervention was measured and compared using a motion capture system (VICON MX System, 16 T20s cameras, $100 \mathrm{~Hz}$, Plug-in gait marker set, Oxford, UK), which was synchronized with EMG and 
sampled at $100 \mathrm{~Hz}$. Sixteen auto-reflective makers were placed in accordance with the plug-in gait marker set bilaterally on the anterior superior iliac spine, posterior superior iliac spine, lower lateral $1 / 3$ surface of the thigh, the flexion-extension axis of the knee, lower lateral $1 / 3$ surface of the shank, lateral malleolus of the ankle, the posterior peak of the calcaneus for the heel, and the lateral second metatarsal bone of the toe. Sagittal angles of bilateral hip, knee and ankle joints were extracted using VICON Nexus software (version 2.2.3). In the pipeline processing of marker position data, the software applies a Woltering filter (Woltring, 1986) which is based on spline fitting for smoothing the marker trajectories.

\subsubsection{Electromyography}

Electromyography was recorded during $10 \mathrm{~m}$ test before the first HAL session and after the last HAL session for prepost comparison, and also in the 4th and 7th HAL sessions immediately before and after using HAL. The 4th session represents the first half of the ten sessions, and the 7th session was extracted as information representative of the second half of the ten sessions.

Six wireless, surface EMG electrodes were placed bilaterally on the muscles relevant to knee joint motion; vastus medialis (VM), hamstrings (HAM), and gastrocnemius (GAS) for the purpose of evaluating knee joint control during gait. The data was sampled using a TrignoTM Lab Wireless electromyography system (Delsys Inc., Boston, MA, USA) at $2 \mathrm{k} \mathrm{Hz}$ synchronized with the motion capture.

\subsection{Data Analysis}

\subsubsection{Joint Angles}

Data on walking speed, cadence and stride length were obtained from clinical assessment. From the motion capture data, joint angle profile of hip, knee and ankle joints were obtained and segmented into step cycles referring to the gait phase detected by toe and heel markers. Swing time and stance time, swing time ratio were obtained. For joint angle analysis, the maximum extension and flexion of hip, knee, ankle joint angles within cycle, and range of motion (ROM) of these joint angles were obtained. The anterior and posterior tilt angles of the pelvis, and toe-lift from the ground before and after the intervention were also compared. Knee joint angle is known to possess double flexion peaks in a gait cycle; one during the stance phase and the other during the swing (Perry and Burnfield, 2010). The first peak after heel landing corresponds to the shock absorption and the second peak corresponds to the flexion during swing phase. The amount of the first flexion was evaluated by the difference between the knee joint angles at the heel landing and the first flexion peak, which is hereafter referred to as the first knee action.

\subsubsection{Electromyography}

Each patient's steps, from first heel-strike to final toe-off were extracted for each leg, and the synchronized electromyography (EMG) tracks and motion data were collected. Hampel filter (time window $=200 ; \sigma$ threshold $=4$ [standard deviations]) was used to remove artifacts from the EMG data, before being rectified and low-passed filtered (4th order zero-lag Butterworth low-pass filter at $6 \mathrm{~Hz}$ to obtain the EMG envelope and then time-normalized and re-sampled to 100-time points.

Regarding muscle activities related to the control of knee joint during gait, deceleration of the angle is known to take place during late swing to prepare for the coming heel strike and the first knee action (Perry and Burnfield, 2010). This phase is characterized as the range between 80 and $100 \%$ of a gait cycle (Levangie and Norkin, 2005) including terminal swing which starts when the tibia crosses the vertical position and ends with the initial contact. The gait phases were extracted referring to the motion capture data which was recorded in synchronization with the EMG. The ratio of EMG values in the $80-100 \%$ of gait cycle to those in the entire cycle was calculated and compared between pre and post HAL intervention. For evaluation of immediate EMG changes in the 4th and 7th sessions, integrated EMG values in the $80-100 \%$ of gait cycle was calculated and compared between just before and after HAL intervention within each of the sessions.

\subsection{Statistical Analysis}

All data are expressed with mean and standard deviation (SD). Comparisons of kinematic and kinetic data between before and after the HAL intervention were made using a paired Wilcoxon signed-rank test for the acute and chronic groups, to compare between baseline measurements and outcomes after all the 10 sessions. Mann-Whitney $U$-Test was used to compare MMT scores between baseline and outcomes. Significance was considered in comparisons with $P<0.05$.

\subsection{Software Tools}

Custom scripts on MATLAB 8.4 and 9.3 (Mathworks Inc., Natick, MA, USA) and R 3.6.0 (The R Foundation for Statistical Computing) were used for data extraction and statistical analysis.

\section{RESULTS}

\subsection{HAL Intervention for Patients at an Acute Stage of Postoperative Gait Disorder} $14.2 \pm 5.7$ steps and $10.8 \pm 2.0$ steps were recorded by the motion capture in $10 \mathrm{~m}$ walking tests before and after the HAL intervention, respectively. Significant improvements were observed in walking speed 24.5 vs. $54.1 \mathrm{~m} / \mathrm{min}(P<0.01)$, and stride length 71.2 vs. $102.5 \mathrm{~cm}(P<0.05)$. Swing time and cadence tended to improve, respectively, by 1.1 vs. $0.8 \mathrm{~s}$ and cadence 35.7 vs. 50.7 cycles/min but statistical significance was not achieved (N.S.) (Table 2). A comparison of the peak of each leg joint angle was used to evaluate the limb movement during the gait (Figure 1). Measurements of hip joint range of movement (ROM) before and after HAL intervention indicated a significant increase in ROM: $34.0^{\circ}$ vs. $43.4^{\circ}(P<0.05)$ (Figure 1A). Knee joint ROM also increased significantly: $42.1^{\circ}$ vs. $56.7^{\circ}(P<0.05)$. The first knee action appeared in the initial stance stage: $3.2^{\circ}$ vs. $5.5^{\circ}(P<0.05)$ (Figure 1B). Ankle joints ROM was $19.9^{\circ}$ vs. $25.5^{\circ}$ (N.S.) (Figure 1C) and the toe-lift was 132.2 vs. $144.8 \mathrm{~mm}$ (N.S.) (Figure 1D). MMT scores of the acute patients showed improvement in hip flexor $(P<0.01)$, knee 
extensor $(P<0.05)$, and ankle dorsi flexor $(P<0.05)$ muscle groups, but not in knee flexor and ankle plantor flexor muscle groups (Table 3 ).

\subsection{HAL Intervention for Patients at a Chronic Stage of Postoperative Gait Disorder}

$16.0 \pm 3.9$ steps and $14.0 \pm 3.5$ steps were recorded by the motion capture in $10 \mathrm{~m}$ walking tests before and after the HAL intervention, respectively. Significant improvements were observed in walking speed 46.5 vs. $53.8 \mathrm{~m} / \mathrm{min}(P<0.01)$,

TABLE 2 | Comparison of gait kinematics between pre and post HAL intervention in acute stage patients.

\begin{tabular}{lccc}
\hline Measurements & Pre HAL & Post HAL & $P$-value \\
\hline Walking speed (meters/minute) & 24.5 & 54.1 & $P<0.01$ \\
Cadence (cycles/minute) & 35.7 & 50.7 & N.S. \\
Stride length (cm) & 71.2 & 102.5 & $P<0.05$ \\
Swing time (seconds) & 1.1 & 0.8 & N.S. \\
ROM of hip joints & $34.0^{\circ}$ & $43.4^{\circ}$ & $P<0.05$ \\
ROM of knee joints & $42.1^{\circ}$ & $56.7^{\circ}$ & $P<0.05$ \\
The first knee action & $3.2^{\circ}$ & $5.5^{\circ}$ & $P<0.05$ \\
ROM of ankle joints & $19.9^{\circ}$ & $25.5^{\circ}$ & N.S. \\
Toe lift (mm) & 132.2 & 144.8 & N.S.
\end{tabular}

cadence 51.7 vs. 54.7 cycles/min $(P<0.05)$, stride length 89.3 vs. $98.0 \mathrm{~cm}(P<0.01)$, and swing time 0.91 vs. $0.92 \mathrm{~s}(P<0.05)$

(Table 4). ROM of hip joint: $36.1^{\circ}$ vs. $40.3^{\circ}(P<0.01)$ has increased and slightly changed to flexion position (Figure 2A). Knee joints ROM was non-significant, and the double knee action has appeared in the initial stance stage: $4.8^{\circ}$ vs. $7.2^{\circ}(P<$ 0.05) (Figure 2B). Ankle joints ROM was $25.5^{\circ}$ vs. $26.7^{\circ}$ (N.S.) (Figure 2C), and it changed to plantar flexion position. This change could have been caused by the displacement of the ROM of hip joint that moved to flexion position. Improvement in toe lift: 109.7 vs. $128.0 \mathrm{~mm}(P<0.01)$ was observed (Figure 2D). Pelvic angle has changed to an anterior tilt angle of $100.4^{\circ}$ vs. $107.2^{\circ}(P<0.05)$ and a posterior tilt angle of $96.0^{\circ}$ vs. $102.5^{\circ}$ $(P<0.05)$ (Figure 2E). MMT scores of the chronic patients

TABLE 3 | Comparison of MMT scores between pre and post HAL intervention in acute stage patients.

\begin{tabular}{lccc}
\hline Muscle group & $\begin{array}{c}\text { Pre HAL } \\
\text { Med [Min-Max] }\end{array}$ & $\begin{array}{c}\text { Post HAL } \\
\text { Med [Min-Max] }\end{array}$ & $\begin{array}{l}\boldsymbol{P} \text {-value } \\
\text { Hip Flex }\end{array}$ \\
Knee Flex & $3[3-4]$ & $4[3-5]$ & $P<0.01$ \\
Knee Ext & $3[3-4]$ & $4[3-4]$ & N.S. \\
Ankle Plant & $3.5[3-5]$ & $4[4-5]$ & $P<0.05$ \\
Ankle Dorsi & $3.5[2-4]$ & $4[3-4]$ & N.S. \\
& $4[3-4]$ & $4[3-5]$ & $P<0.05$
\end{tabular}

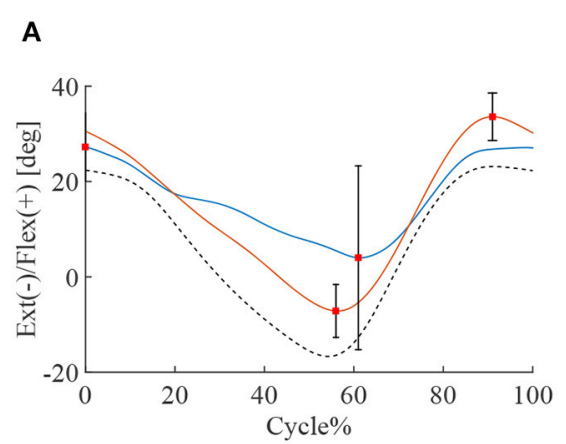

B
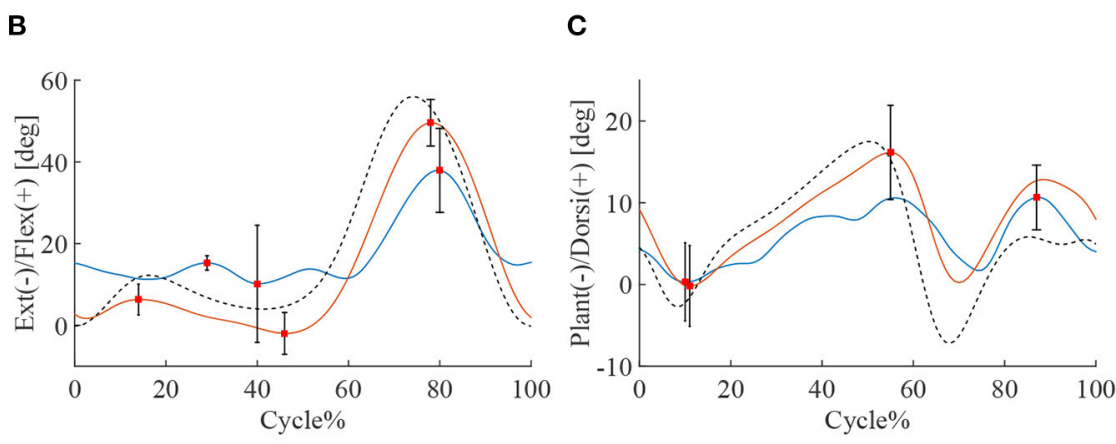

D
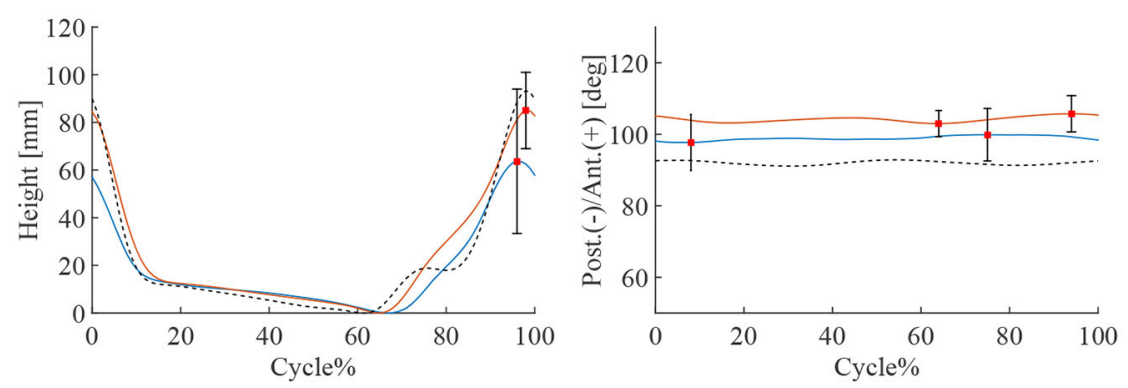

FIGURE 1 | Gait kinematics of acute group patients. Blue line is the mean of patients' joint angle before HAL intervention, the red line is post HAL intervention, and the dotted line is gait analysis plot for healthy subjects. The percentage of walking cycle represents the normalized time. (A) Hip joint. (B) Knee joint. (C) Ankle joint. (D) Toe lift. (E) Pelvis. 
showed improvement in hip flexor muscle group $(P<0.05)$, but not in the other lower limb muscle groups (Table 5).

\subsection{Comparison of Joint Angles With Healthy Subjects}

Peak joint angles of acute and chronic stage patients, both pre and post HAL intervention, was compared with healthy volunteers (Figure 3). Range of motion (ROM) of knee joint showed a significant difference between patients of both acute and chronic groups and healthy subjects before HAL intervention. However, these differences have disappeared after the HAL intervention in both acute and chronic phases (Figure 3,

TABLE 4 | Comparison of gait kinematics between pre and post HAL intervention in chronic stage patients.

\begin{tabular}{lccc}
\hline Measurements & Pre HAL & Post HAL & $P$ value \\
\hline Walking speed (meters/minute) & 46.5 & 53.8 & $P<0.01$ \\
Cadence (steps/minute) & 51.7 & 54.7 & $P<0.05$ \\
Stride length (cm) & 89.3 & 98.0 & $P<0.01$ \\
Swing time (seconds) & 0.91 & 0.92 & $P<0.05$ \\
ROM of hip joints & $36.1^{\circ}$ & $40.3^{\circ}$ & $P<0.05$ \\
ROM of knee joints & $46.9^{\circ}$ & $51.9^{\circ}$ & N.S. \\
The first knee action & $4.8^{\circ}$ & $7.2^{\circ}$ & $P<0.05$ \\
ROM of ankle joints & $25.5^{\circ}$ & $26.7^{\circ}$ & N.S. \\
Toe lift (mm) & 109.7 & 128.0 & $P<0.01$
\end{tabular}

Knee ROM). Table 6 shows RMS (root mean squared) difference of gait kinematics parameters of acute and chronic stage patients, pre and post HAL intervention, in comparison to healthy subjects. It also shows the change of RMS between pre and post.

\subsection{EMG Analysis of Double Knee Action}

For acute patients, the ratio of EMG values of HAM in the late to terminal swing phase (80-100\% of normalized gait cycle) against the full cycle was significantly higher in post-HAL intervention $(P=0.0137)$ (Figure 4, Acute). However, EMG values of VM $(P=0.3804)$ and GAS $(P=0.5693)$ were not significantly different. Chronic patients did not show significant differences; VM $(P=0.1330), \operatorname{HAM}(P=0.6791)$ and GAS $(P=0.6165)$ (Figure 4, Chronic).

TABLE 5 | Comparison of MMT scores between pre and post HAL intervention in chronic stage patients.

\begin{tabular}{lccc}
\hline Muscle Group & $\begin{array}{c}\text { Pre HAL } \\
\text { Med [Min-Max] }\end{array}$ & $\begin{array}{c}\text { Post HAL } \\
\text { Med [Min-Max] }\end{array}$ & $\boldsymbol{P}$ value \\
\hline Hip Flex & $4[3-5]$ & $4[3-5]$ & $P<0.05$ \\
Knee Flex & $4[3-5]$ & $5[3-5]$ & N.S. \\
Knee Ext & $4[4-5]$ & $5[3-5]$ & N.S. \\
Ankle Plant & $4[2-5]$ & $4[2-5]$ & N.S. \\
Ankle Dorsi & $4[1-5]$ & $5[1-5]$ & N.S.
\end{tabular}

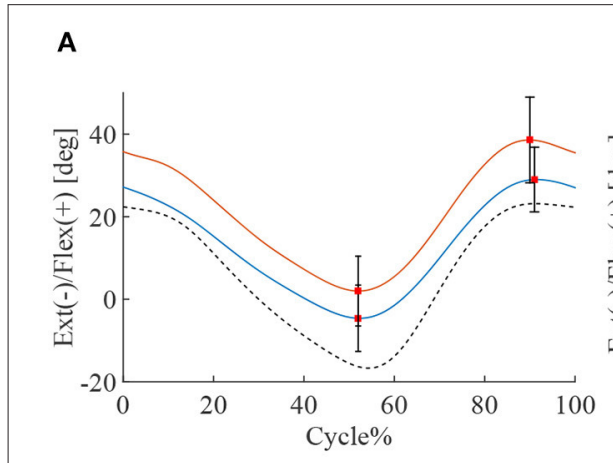

B

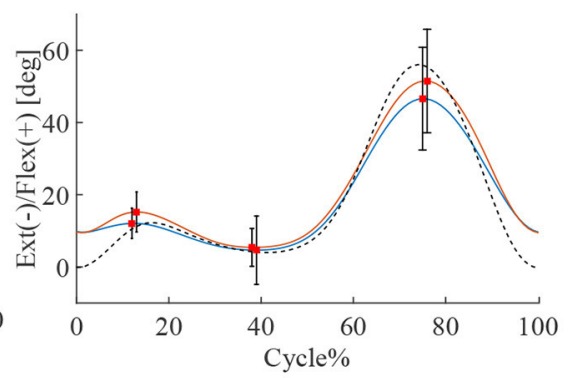

C

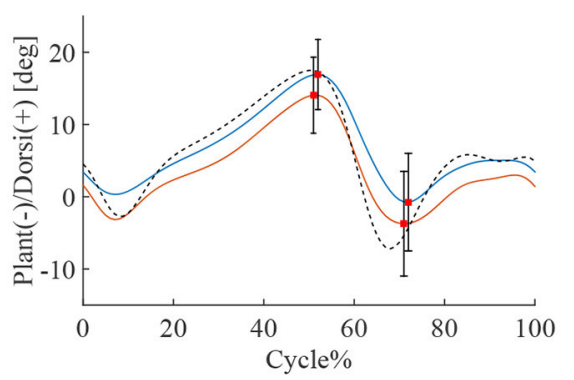

D

E
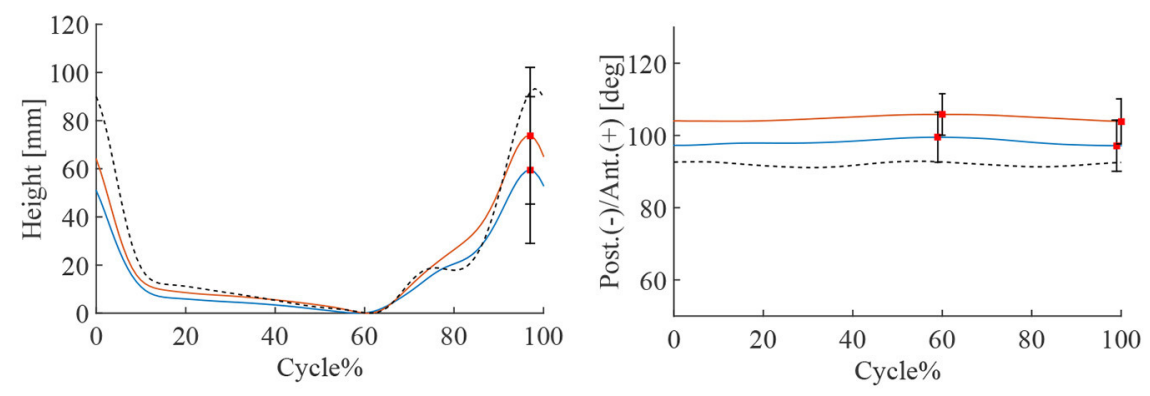

FIGURE 2 | Gait kinematics of chronic stage patients. Blue line is the mean of patients' joint angle before HAL intervention, the red line is post HAL intervention, and the dotted line is gait analysis plot for percentage of walking cycle represents the normalized time. (A) Hip joint. (B) Knee joint. (C) Ankle joint. (D) Toe lift. (E) Pelvis. 

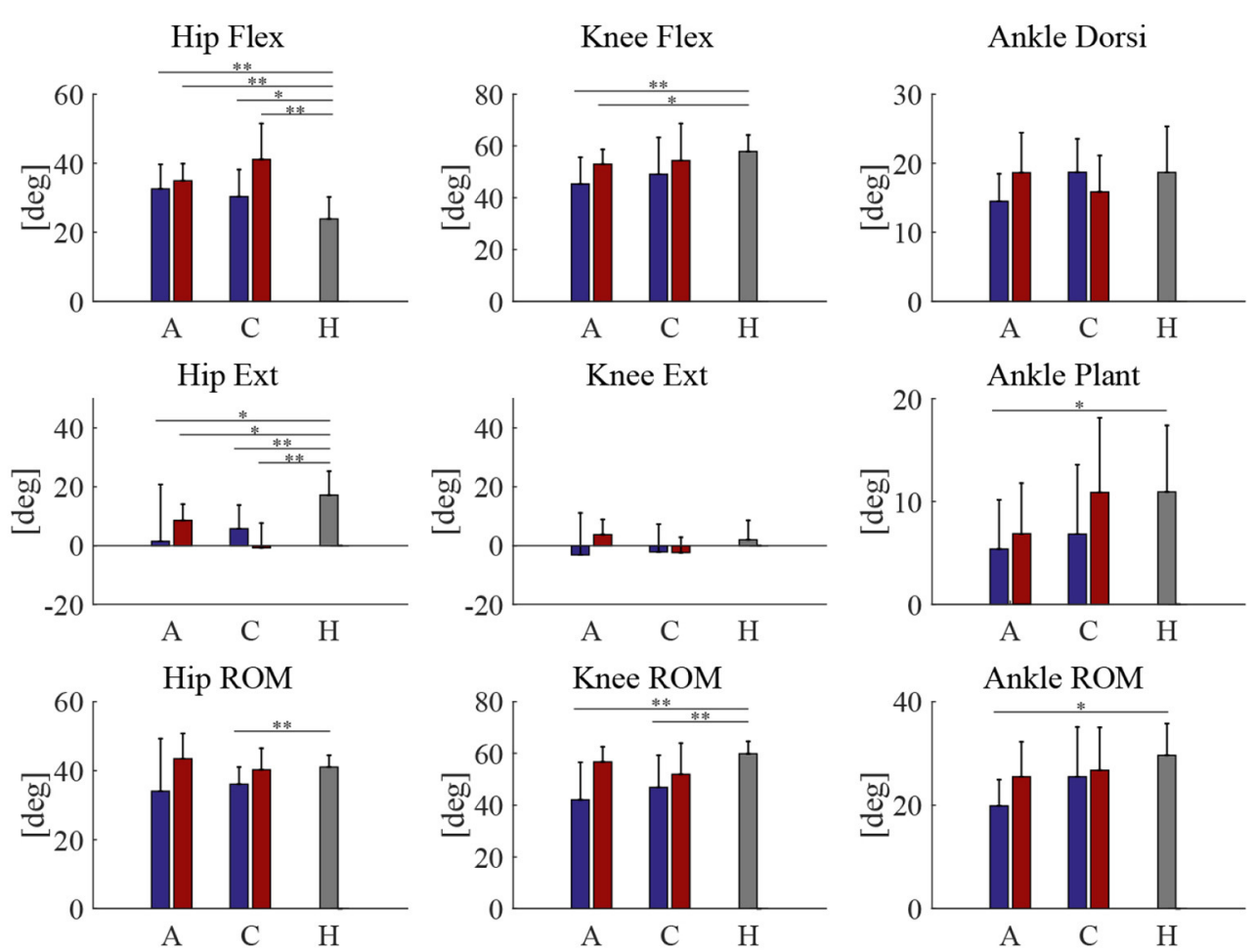

FIGURE $3 \mid$ Peak joint angles | A, C, H is stands for acute, chronic, and healthy, respectively. Blue and red bars represent pre and post HAL intervention, respectively. ${ }^{* *} P<0.01,{ }^{*} P<0.05$, and no sign means $P>0.05$.

\subsection{Analysis of Immediate Muscle Activity Changes}

In the analysis of immediate gait changes in the 4th and 7 th sessions (Figures 5, 6 and Tables 7, 8), walking speed, cadence, stride length or toe lift did not show significant difference in both of acute and chronic stage patients, except that only toe lift became higher at the 4th session in the chronic stage patients $(P<0.05)$. On the other hand, EMG analysis of late to terminal swing phase showed that activation of VM decreased in the 4th HAL session of the acute patients $(P<0.01)$ and also tended to decrease at the 7th session of the acute and at the 4th session of chronic patients. (N.S., 4th Chronic $P=0.091$, 7th Acute $P$ $=0.077)$. Activation of GAS decreased in the 7 th session of the chronic patients $(P<0.01)$ and tended to decrease at the 4th HAL session of both acute and chronic patients (N.S., 4th Acute $P=0.064,4$ th Chronic $P=0.058)$ (Tables 5, 6).

\section{DISCUSSION}

Improvements were observed in the form of faster walking speed, higher cadence and longer strides with smooth foot clearance and shortened swing time in both acute and chronic phase patients. Increased ROM of hip and knee joints contributed to longer strides. First knee action which is typical for a healthy gait has appeared after the HAL intervention, enabling the patients to use knee as a cushion at initial contact. Even without the significant changes in ankle joint, there were improvement in toe lift. It was achieved by the combination of enhanced ROM of hip and knee joints. In chronic patients, ROM of the hip joint slightly shifted to flexion position, while the ankle joint's ROM shifted to plantar flexion position. Regarding the toe lift, the geometrical relationship is that flexion of the hip improves the toe lift, while plantar flexion of the ankle decreases the toe lift. Since the data shows that the toe lift improved after HAL intervention, the combination of these two shifting resulted in higher toe lifting. This is beneficial in daily practical walking, in that it contributes to prevent stumbling. When the patients walked with HAL, we observed that they are more likely to walk with their legs up high. Chronic patients who were able to walk in the first stage were especially prone to gait accentuation, and this series of improvements in ROM made them raise their toes higher.

Anterior shift of the pelvic orientation was observed in both the acute and the chronic patients. We consider that this was caused by the HAL's mechanical structure, which tends to constrain pelvic posture. With respect to angular momentum, pelvic rotation and its stability is important in high velocity walking rather than in slower walking (below $3 \mathrm{~km} / \mathrm{h}$ ), since legs and arms play more important roles in faster walking (Bruijn et al., 2008). So that this slight pelvic angle change might not be a problem in low velocity walk during rehabilitation. It was also discussed that knee flexion amount contributes to controlling the center of mass more than 
TABLE 6 | RMS (root mean squared) difference of gait kinematics of acute and chronic stage patients, pre and post intervention, compared to healthy subjects, and its changes between pre and post.

\begin{tabular}{|c|c|c|c|c|c|c|}
\hline \multirow[b]{2}{*}{ Parameters } & \multicolumn{3}{|c|}{ Acute } & \multicolumn{3}{|c|}{ Chronic } \\
\hline & Pre & Post & Pre - post & Pre & Post & Pre - post \\
\hline Walking Speed (m/min) & 43.0 & 18.6 & 24.4 & 23.6 & 18.7 & 4.93 \\
\hline Cadence (cycles/m) & 17.8 & 8.6 & 9.2 & 7.1 & 7.3 & -0.24 \\
\hline Stride Length (cm) & 62.0 & 26.7 & 35.4 & 40.0 & 33.4 & 6.53 \\
\hline Swing Ratio (\%) & 14.9 & 12.0 & 2.90 & 8.99 & 3.56 & 5.43 \\
\hline Swing Duration (s) & 0.24 & 0.11 & 0.14 & 0.074 & 0.11 & -0.038 \\
\hline Stance Duration (s) & 0.437 & 0.253 & 0.184 & 0.155 & 0.0725 & 0.0825 \\
\hline Hip Max Flex (deg) & 11.0 & 12.0 & -0.968 & 9.94 & 19.9 & -9.97 \\
\hline Hip Max Ext (deg) & 24.1 & 10.1 & 14.0 & 13.8 & 19.8 & -5.93 \\
\hline Hip ROM (deg) & 16.1 & 7.37 & 8.69 & 6.91 & 5.97 & 0.935 \\
\hline Knee Max Flex (deg) & 15.9 & 7.30 & 8.58 & 16.3 & 14.2 & 2.03 \\
\hline Knee Max Ext (deg) & 14.6 & 5.17 & 9.38 & 10.0 & 6.71 & 3.34 \\
\hline Knee ROM (deg) & 22.4 & 6.33 & 16.08 & 17.7 & 14.0 & 3.64 \\
\hline Ankle Max Dors (deg) & 5.62 & 5.47 & 0.149 & 4.67 & 5.80 & -1.12 \\
\hline Ankle Max Plant (deg) & 7.16 & 6.24 & 0.925 & 7.70 & 6.98 & 0.717 \\
\hline Ankle ROM (deg) & 10.8 & 7.62 & 3.22 & 10.1 & 8.47 & 1.66 \\
\hline Toe Max Height (mm) & 30.6 & 15.3 & 15.2 & 44.1 & 31.1 & 13.1 \\
\hline Pelvis Max Ant. (deg) & 9.99 & 13.6 & -3.66 & 9.34 & 14.6 & -5.23 \\
\hline Pelvis Max Post. (deg) & 9.21 & 12.3 & -3.07 & 8.62 & 13.5 & -4.83 \\
\hline Pelvis ROM (deg) & 2.52 & 2.20 & 0.328 & 2.40 & 2.20 & 0.195 \\
\hline Double Knee Action (deg) & 9.81 & 8.18 & 1.63 & 8.99 & 7.74 & 1.25 \\
\hline
\end{tabular}

Bold numbers indicate positive pre-post RMS, meaning closer to healthy values at post HAL assessment than at pre HAL.

pelvic rotation (Della Croce et al., 2001; Kerrigan et al., 2001). Therefore, we believe that the influence of pelvic rotation was small compared to the merit of gaining double knee action. It is left for future studies to improve the pelvis harness to achieve some level of rotational movement while providing enough support.

In a healthy gait, there are two episodes of knee flexion during gait; the first knee flexion takes place at the initial contact to loading response phase and the second knee flexion takes place during the period from terminal stance to pre-swing phase. This double knee action is important for a healthy gait, especially the first knee action is essential for shock absorption just after landing (Farrokhi et al., 2015). Knee hyper-extension, or genu recurvatum is a common gait issue, which is caused by poor muscle activation. It is observed in $40-60 \%$ of poststroke patients (Cooper et al., 2012). Patients' pathological gait with severe myelopathy is also characterized with knee hyperextension in the stance phase (Maezawa et al., 2001). This knee hypertension is developed to compensate their weaknesses in calf and quadriceps muscles, and remained even after 6 months when their velocity had improved (Mulroy et al., 2003). The knee hypertension is also prevalent among the obese population. The more extended leg may allow them to reduce vasti muscle activity and joint forces (DeVita and Hortobágyi, 2003). Their weakness in gluteus medius muscle may cause increased hip adduction during stance and require the stance leg to be extended more for the swing leg clearance (Haight et al., 2014). Once this kind of pathological gait is developed, the patients are more likely to develop knee hypertension and end up having a locomotive syndrome or requiring another surgical intervention (Bleyenheuft et al., 2010; Mao et al., 2015).

Prolonged hyper-extension or rapid extension may cause fatigue in the muscles responsible for stability, and chronic over tension causes weathering, degeneration and pain in the soft tissues that support the knee joint. The patients developed double knee action after the HAL intervention instead of resulting in knee hypertension, which led to smoother and closer to healthy gait.

EMG of VM, HAM, and GAS was analyzed to show that the gait improvement was achieved not only from a kinematical view, but also from a physiological perspective. The muscles that mainly control knee movement are HAM, GAS, and VM: HAM and GAS for knee flexion; VM for knee extension. The combination of those muscle's movement enables smooth knee action. The first knee action from initial contact to mid stance is important for shock absorption. HAM starts to regulate the rate of knee joint extension at late swing phase $(87-100 \%$ of gait cycle) by efferent decelerating (Perry and Burnfield, 2010). There are several possible mechanisms to explain knee flexion limitation at the load response period. The complete lack of knee flexion in load-responsive period is often caused by an intentional compensatory movement to reduce the demand on quadriceps in case of weak quadriceps femoris (Mulroy et al., 2003). Possible causes of first knee action loss include weakness of the quadriceps femoris (Perry and Burnfield, 2010). Loss of first knee action reduces normal shock absorption and places less demand on the quadriceps.

In the acute phase, we observed EMG improvement in timing of muscle activity of HAM (Figure 4, Acute, HAM). HAM's improvement of timing in late swing phase is important for braking the swing, that suggests the preparation for flexion in initial contact. Due to the lack of accurate training, patients tend to use knee hypertension to compensate their muscle weakness. Without using HAM in swing phase, they keep extending their knees and tend to have difficulty landing with flexed knee at initial contact. After the HAL intervention, their breaking in swing phase got better.

In the chronic phase patients, there were no apparent changes in EMG of the three muscles after HAL intervention even though improvement of double knee action was observed in their joint angles. The joint angle analysis showed that the chronic patients had small first knee actions before the HAL intervention. Even they improved its flexion amount, this small improvement in joint angles were not obvious in EMG. It is also difficult to change their long-acquired compensatory gait motion over a comparatively short period of training once they got used to it. It has been shown that coordinated motor engrams need a tremendous amount of repeated training (Kottke et al., 1978). After formation of a pathological gait, the old engrams need to be inhibited, and it takes more time to relearn the movements. Longer periods of training, or early phase intervention might be required to modify their compensatory gait. Thus, it is important to re-learn healthy gait at the early stage of recovery with minimal 


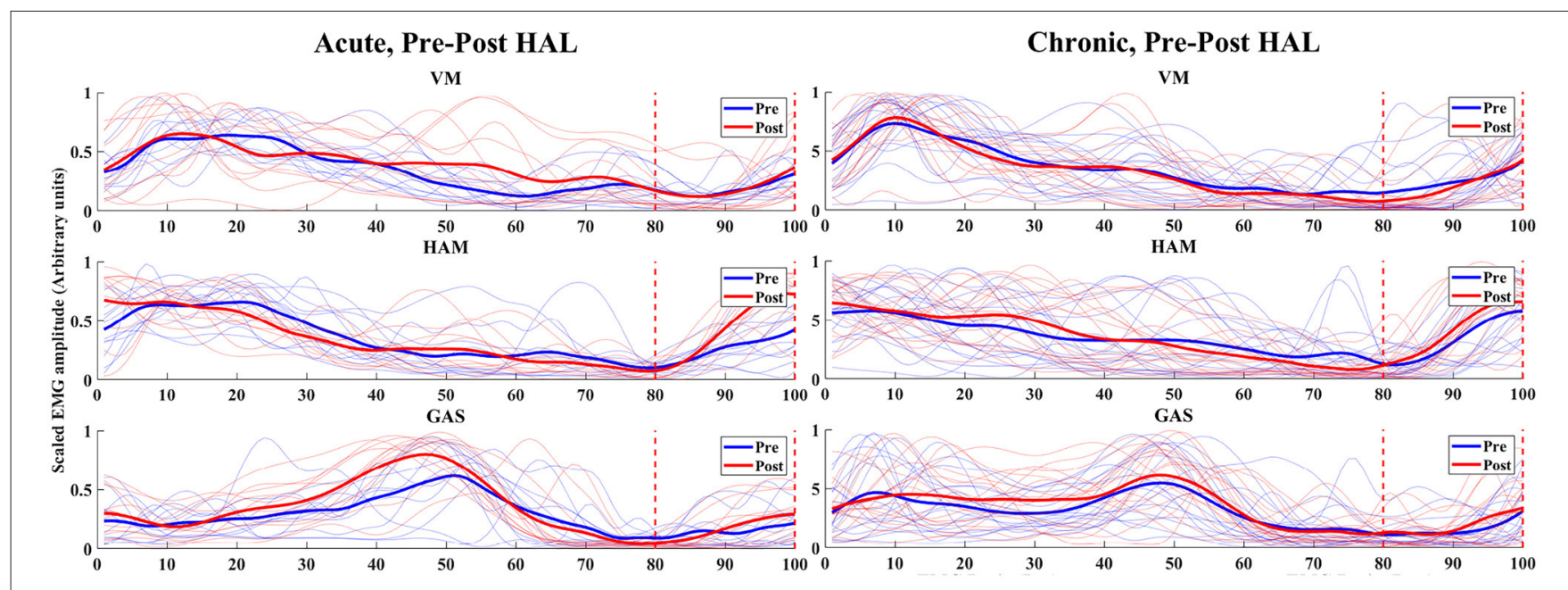

FIGURE 4 | EMG (VM, HAM, and GAS) of acute and chronic stage patients, pre and post HAL intervention - The normalized time correspond to the percentage of walking cycle. The blue and red lines shows pre and post HAL intervention plots of each subject's EMG, respectively. The solid blue and red lines are mean plots of all subject's pre and post HAL intervention in the group, respectively. Eighty percent to one hundred percent of gait cycle and the late swing phase to terminal swing phase was estimated using synchronized motion data.

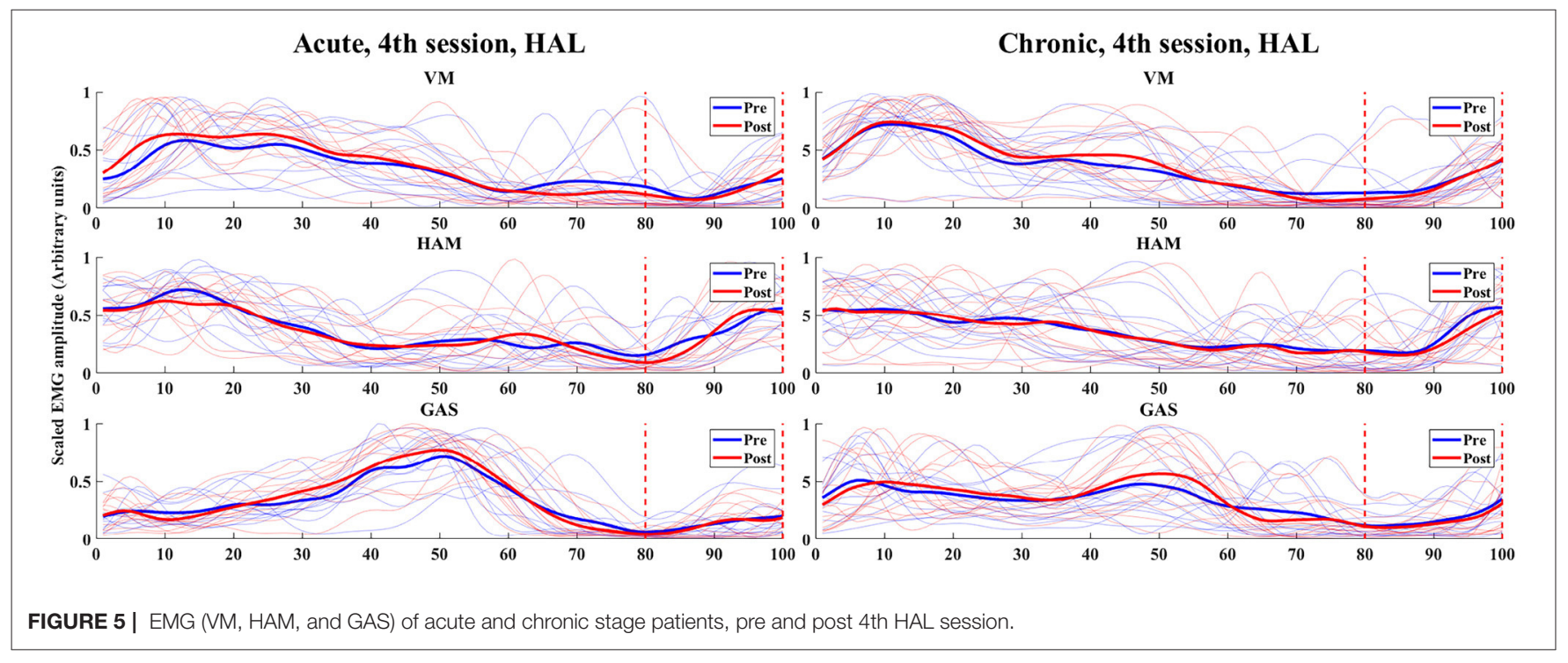

compensation, particularly for those presents an asymmetric locomotion (Barbeau, 2003).

We observed improvements in walking speed, cadence, and stride length after the HAL training in both acute and chronic stage of postoperative OPLL patients. However, it is difficult to distinguish whether this recovery is achieved by the HAL intervention or solely in conjunction with patient's recovery of muscle strength. To clarify this, MMT scores of the participants before and after HAL intervention were compared. MMT scores of the acute patients showed improvement in hip flexor, knee extensor, and ankle dorsi flexor (Table 3). These improvements were mostly correlated with improvement in joint angle ROMs (Table 2). MMT scores of chronic patients showed improvement only in the hip flexor muscle (Table 5) which is correlated with significant improvement in ROM of hip joints (Table 4), but not in the other lower limb muscle groups even though their double knee action was improved. However, it is reported that the activity level of most muscles in normal gait of healthy people is equivalent to MMT 3 (Perry et al., 1986). Although muscle strength is necessary to achieve gait, muscle strength does not play the main role in gait improvement once basic necessary muscle strength is acquired. In this sense, learning to control timing and coordination of related muscles during gait is needed to achieve smooth and effective gait, besides the capability of maximum exertion of each single muscle.

As immediate change after HAL therapy, $10 \mathrm{~m}$ test before and after each 4 th and 7 th session was compared. Except the 7 th HAL session of chronic patients, EMG of VM tended to decrease 


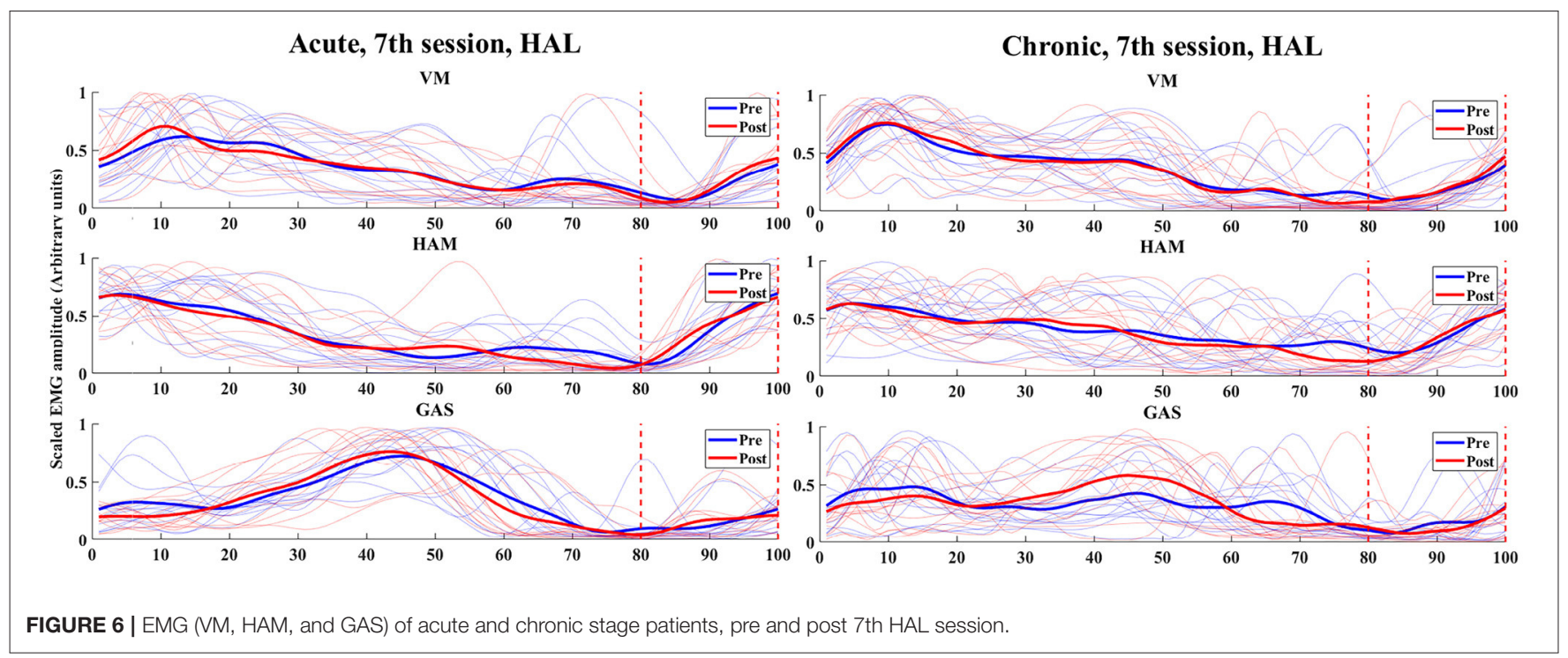

TABLE 7 | Comparison of integrated EMG of the late to terminal swing phase during $10 \mathrm{~m}$ walking test immediately before and after HAL intervention at the 4th HAL session, in acute and chronic stage patients.

\begin{tabular}{lcccccc}
\hline $\begin{array}{l}\text { 4th session } \\
\text { Parameters }\end{array}$ & Pre & $\begin{array}{c}\text { Acute } \\
\text { Post }\end{array}$ & $\boldsymbol{P}$-value & Pre & Post & P-value \\
\hline Walking speed $(\mathrm{m} / \mathrm{min})$ & 40.2 & 40.1 & 0.964 & 42.9 & 42.8 & 0.936 \\
Cadence (cycles/min) & 41.0 & 42.5 & 0.114 & 47.9 & 45.7 & 0.380 \\
Stride length (cm) & 84.6 & 84.1 & 0.833 & 75.4 & 68.6 & 0.672 \\
Toe lift (mm) & 93.8 & 99.3 & 0.239 & 76.5 & 82.7 & $\mathbf{0 . 0 1 3}$ \\
VM (mV) & 0.1743 & 0.1169 & $\mathbf{0 . 0 0 0}$ & 0.3759 & 0.2290 & 0.091 \\
HAM (mV) & 0.2457 & 0.2144 & 0.380 & 0.2253 & 0.1858 & 0.469 \\
GAS (mV) & 0.3000 & 0.1087 & 0.064 & 0.2082 & 0.1630 & 0.058 \\
\hline
\end{tabular}

Bold numbers mean $P<0.05$ or $P<0.01$.

TABLE 8 | Comparison of integrated EMG of late to terminal swing phase during $10 \mathrm{~m}$ walking test immediately before and after $\mathrm{HAL}$ intervention at the $7 \mathrm{th} \mathrm{HAL}$ session, in acute and chronic stage patients.

\begin{tabular}{lcccccc}
\hline 7th session & \multicolumn{3}{c}{ Acute } & & \multicolumn{3}{c}{ Chronic } \\
Parameters & Pre & Post & P value & Pre & Post & P value \\
\hline walking speed (m/min) & 53.8 & 51.3 & 0.101 & 43.5 & 40.1 & 0.378 \\
cadence (cycles/min) & 49.5 & 47.5 & 0.053 & 45.2 & 46.2 & 0.289 \\
stride length (cm) & 92.7 & 93.9 & 0.584 & 83.5 & 82.5 & 0.646 \\
toe lift (mm) & 105.5 & 105.7 & 0.984 & 82.9 & 86.9 & 0.294 \\
VM (mV) & 0.1694 & 0.1305 & 0.077 & 0.9187 & 0.2837 & 0.268 \\
HAM (mV) & 0.1678 & 0.1711 & 0.791 & 0.2705 & 0.2446 & 0.578 \\
GAS (mV) & 0.1363 & 0.1123 & 0.176 & 0.3191 & 0.2139 & $\mathbf{0 . 0 0 9}$ \\
\hline
\end{tabular}

Bold numbers mean that $P<0.05$ or $P<0.01$.

after HAL session during late to terminal phase, which indicates less knee extension. Also, EMG of GAS tended to decrease after HAL session except 7 th acute patients, which indicates less ankle plantar flexion during late to terminal phase (Tables 7, 8). Both changes above were helpful to decrease hyper-knee extension. At the same time, their walking speed before and after the session did not change. This indicates that they were learning efficient gait without causing knee hypertension by HAL therapy. Considering also the results of MMT, it could be said that gait improvement by HAL includes improvement of coordinated gait control, not only muscle strengthening. It is noted that simple muscle strengthening exercises for a single or few muscles do not result in the acquisition of motor functions with functional reorganization of the brain (Plautz et al., 2000).

This study has limitations of not including a control group of OPLL patients undergoing conventional rehabilitation to be compared with patients after HAL intervention. Since OPLL is designated as an intractable disease in Japan, the number of patients is about $0.02 \%$ of the population (Matsunaga and Sakou, 2006), indicating difficulty establishing a control group. The number of severe paralysis patients who need assistance for sitting, standing, and walking is enrolled in our research, therefore the number of patients was even smaller. Clinical trial needs such patient control, which is left for future studies. Additionally, long-term prognosis was not investigated in our study. So far, a case report (Kubota et al., 2019) describes a patient with severe spinal cord disorder after OPLL who improved gait after HAL intervention, and the improved gait was maintained at the time of follow up assessment 1 year after the HAL intervention. To formalize a cohort study to track prognosis of the patients who underwent HAL treatment, it would be necessary to control the patients' daily activities to some extent in terms of the amount, frequency and strength of physical activities. It is left for future studies, where assessment of long-term prognosis using multivariate analysis after HAL intervention would be desired.

Muscle synergy analysis is reported as a useful tool for analyzing improved lateral symmetry of gait in stroke patients after HAL intervention (Tan et al., 2018). OPLL is not technically considered as causing lateral asymmetry, but patients 
usually develop asymmetrical gait since they tend to put more weight on a stronger leg and end up developing asymmetrical gait. In this sense, analysis of muscle synergy before and after HAL intervention may be a useful tool to evaluate muscle activity of OPLL patients (Kadone et al., 2020) after HAL intervention.

HAL is made to build a feedback loop like a normal voluntary movement, where movement is generated from voluntary neural and muscular activity and sensory input is provided. It was also shown that the awareness of voluntary actions is involved in the improvement of motor skills (Matsumiya, 2021). Though kinematic improvements were observed in this research, it is also important to study and clarify the neural mechanisms with which the HAL takes effect on the central nervous system. In future studies, radiological and neurophysiological assessments would help to further clarify these points.

Learning healthy gait instead of compensation gait is more desirable and it is expected in rehabilitation using medical exoskeletons which are focused on maximizing functional independence, balance, and restoring gait function. Those taskspecific over-ground training is more helpful and supplement conventional rehabilitation (Esquenazi et al., 2017). The analysis presented in this study could help to establish indexes and scales to evaluate quality of gait so that the patients can regain healthier gait that can last long.

\section{CONCLUSION}

We measured and assessed the gait before and after the HAL intervention for postoperative OPLL patients with gait impairment in acute and chronic stage of post decompression operation. The patients gained faster walking speed, longer strides and smoother gait after the HAL intervention. ROM of hip, knee, and ankle joints have improved significantly in acute phase patients. All the joint angles except ankle joint have improved significantly in chronic patients. Importantly, double knee action of initial contact to loading response phase improved and knee hypertension was reduced, enabling the patients to gain closer to healthy gait. Also, all those improvement was not only made by muscle strengthening but also by learning coordinated gait control.

It is still true that conventional rehabilitation training is also efficient for patients. However, it would be difficult to repeat the exercises with the healthy gait pattern from the beginning to form a correct engram. It is possible for HAL to intervene from the acute stage even when walking is difficult without support, and to realize repetitive training of correct gait. During this period, it also includes muscle strengthening through natural recovery, but the difference is that it is not single muscle strengthening training, but functional motor learning of gait.

Staying in low ADL with less mobility or altered gait mechanics with increased pain, decreased muscle mass, proprioception deficits would cause other chronic diseases including osteoporosis, obesity, hyperglycemia and cardiopulmonary complications. The HAL can provide repeatable, standard, sustainable, and voluntary training and the current research reports the feasibility and effectiveness of HAL for functional recovery in rehabilitation use for multiple neurological disorders patients with gait disturbance after spinal cord diseases and cerebral vascular diseases.

\section{DATA AVAILABILITY STATEMENT}

The raw data supporting the conclusions of this article will be made available by the authors, without undue reservation.

\section{ETHICS STATEMENT}

The studies involving human participants were reviewed and approved by The University of Tsukuba Hospital Ethics Committee. The patients/participants provided their written informed consent to participate in this study.

\section{AUTHOR CONTRIBUTIONS}

SE and HK collected, analyzed, and interpreted the data. SE wrote and drafted and $\mathrm{HK}$ edited the manuscript. SK administered HAL therapy and collected clinical scores. TA diagnosed and operated all the patients from acute group. YSh supported the HAL therapy. CT assisted the data analysis. $\mathrm{KM}, \mathrm{YH}$, and $\mathrm{MK}$ provided important comments on the planning and implementation of HAL treatment. YSa originally developed the robot suit HAL and conceived the idea of HAL therapy. KS provided essential insights for the analysis. MY operated all patients from chronic group, developed HAL therapy for OPLL patients, and organized the study. All authors made critical revisions of the manuscript and approved the final version.

\section{FUNDING}

This study was supported by the Industrial Disease Clinical Research Grant of the Ministry of Health, Labour and Welfare, Japan (14060101-01).

\section{ACKNOWLEDGMENTS}

We are grateful to Mr. Thomas D. Mayers, Medical English Communications Center, University of Tsukuba, for grammatical revision of this manuscript. We are also grateful to Ms. Mayuko Sakamaki and Ms. Yumiko Ito of the Center for Innovative Medicine and Engineering (CIME) of the University of Tsukuba Hospital for their excellent technical assistance. 


\section{REFERENCES}

Aach, M., Cruciger, O., Sczesny-Kaiser, M., Höffken, O., Meindl, R. C., Tegenthoff, M., et al. (2014). Voluntary driven exoskeleton as a new tool for rehabilitation in chronic spinal cord injury: a pilot study. Spine J. 14, 2847-2853. doi: 10.1016/j.spinee.2014.03.042

Barbeau, H. (2003). Locomotor training in neurorehabilitation: emerging rehabilitation concepts. Neurorehabil. Neural Repair 17, 3-11. doi: $10.1177 / 0888439002250442$

Bleyenheuft, C., Bleyenheuft, Y., Hanson, P., and Deltombe, T. (2010). Treatment of genu recurvatum in hemiparetic adult patients: a systematic literature review. Ann. Phys. Rehabil. Med. 53, 189-199. doi: 10.1016/j.rehab.2010.01.001

Bruijn, S. M., Meijer, O. G., van Dieën, J. H., Kingma, I., and Lamoth, C. J. (2008). Coordination of leg swing, thorax rotations, and pelvis rotations during gait: The organisation of total body angular momentum. Gait Post. 27, 455-462. doi: 10.1016/j.gaitpost.2007.05.017

Committee for Clinical Guidelines of the Japanese Orthopaedic Association (2011). Guideline for the Treatment of Ossification of the Posterior Longitudinal Ligament of the Cervical Spine 2011. Nanjiang.

Cooper, A., Alghamdi, G. A., Alghamdi, M. A., Altowaijri, A., and Richardson, S. (2012). The relationship of lower limb muscle strength and knee joint hyperextension during the stance phase of gait in hemiparetic stroke patients. Physiother. Res. Int. 17, 150-156. doi: 10.1002/pri.528

Della Croce, U., Riley, P. O., Lelas, J. L., and Kerrigan, D. C. (2001). A refined view of the determinants of gait. Gait Post. 14, 79-84. doi: 10.1016/S0966-6362(01)00128-X

DeVita, P., and Hortobágyi, T. (2003). Obesity is not associated with increased knee joint torque and power during level walking. J. Biomech. 36, 1355-1362. doi: 10.1016/S0021-9290(03)00119-2

Dobkin, B., Apple, D., Barbeau, H., Basso, M., Behrman, A., Deforge, D., et al. (2006). Weight-supported treadmill vs over-ground training for walking after acute incomplete sci. Neurology 66, 484-493. doi: $10.1212 / 01 . w n l .0000202600 .72018 .39$

Epstein, N. (2002). Diagnosis and surgical management of cervical ossification of the posterior longitudinal ligament. Spine J. 2, 436-449. doi: 10.1016/S1529-9430(02)00394-7

Epstein, N. (2014). What you need to know about ossification of the posterior longitudinal ligament to optimize cervical spine surgery: a review. Surg. Neurol. Int. 5(Suppl 3):S93-S118. doi: 10.4103/2152-7806.130696

Esquenazi, A., Talaty, M., and Jayaraman, A. (2017). Powered exoskeletons for walking assistance in persons with central nervous system injuries: a narrative review. PM R 9, 46-62. doi: 10.1016/j.pmrj.2016.07.534

Farrokhi, S., O'Connell, M., Gil, A. B., Sparto, P. J., and Fitzgerald, G. K. (2015). Altered gait characteristics in individuals with knee osteoarthritis and selfreported knee instability. J. Orthopaed. Sports Phys. Therapy 45, 351-359. doi: 10.2519/jospt.2015.5540

Fehlings, M. G., Ibrahim, A., Tetreault, L., Albanese, V., Alvarado, M., Arnold, P., et al. (2015). A global perspective on the outcomes of surgical decompression in patients with cervical spondylotic myelopathy: results from the prospective multicenter aospine international study on 479 patients. Spine 40, 1322-1328. doi: 10.1097/BRS.0000000000000988

Fehlings, M. G., Tetreault, L. A., Riew, K. D., Middleton, J. W., and Wang, J. C. (2017). A Clinical practice guideline for the management of degenerative cervical myelopathy: introduction, rationale, and scope. Glob. Spine J. 7(3_Supplement), 21S-27S. doi: 10.1177/2192568217703088

Fujii, K., Abe, T., Kubota, S., Marushima, A., Kawamoto, H., Ueno, T., et al. (2017). The voluntary driven exoskeleton Hybrid Assistive Limb (HAL) for postoperative training of thoracic ossification of the posterior longitudinal ligament: a case report. J. Spinal Cord Med. 40, 361-367. doi: $10.1080 / 10790268.2016 .1142056$

Haight, D. J., Lerner, Z. F., Board, W. J., and Browning, R. C. (2014). A comparison of slow, uphill and fast, level walking on lower extremity biomechanics and tibiofemoral joint loading in obese and nonobese adults. J. Orthopaed. Res. 32, 324-330. doi: 10.1002/jor.22497

Hidler, J., Nichols, D., Pelliccio, M., Brady, K., Campbell, D. D., Kahn, J. H., et al. (2009). Multicenter randomized clinical trial evaluating the effectiveness of the Lokomat in subacute stroke. Neurorehabil. Neural Repair 23, 5-13. doi: $10.1177 / 1545968308326632$
Huang, V. S., and Krakauer, J. W. (2009). Robotic neurorehabilitation: a computational motor learning perspective. J. Neuroeng. Rehabil. 6, 1-13. doi: 10.1186/1743-0003-6-5

Ikumi, A., Kubota, S., Shimizu, Y., Kadone, H., Marushima, A., Ueno, T., et al. (2017). Decrease of spasticity after hybrid assistive limb $\AA$ training for a patient with C4 quadriplegia due to chronic SCI. J. Spinal Cord Med. 40, 573-578. doi: 10.1080/10790268.2016.1225913

Kadone, H., Kubota, S., Abe, T., Noguchi, H., Miura, K., Koda, M., et al. (2020). Muscular activity modulation during post-operative walking with hybrid assistive limb (HAL) in a patient with thoracic myelopathy due to ossification of posterior longitudinal ligament: A Case Report. Front. Neurol. 11:102. doi: 10.3389 /fneur.2020.00102

Kasai, R., and Takeda, S. (2016). The effect of a hybrid assistive limb $\AA$ on sitto-stand and standing patterns of stroke patients. J. Phys. Therapy Sci. 28, 1786-1790. doi: 10.1589/jpts.2016.1786

Kawamoto, H., Kamibayashi, K., Nakata, Y., Yamawaki, K., Ariyasu, R., Sankai, Y., et al. (2013). Pilot study of locomotion improvement using hybrid assistive limb in chronic stroke patients. BMC Neurol. 13:141. doi: 10.1186/1471-2377-13-141

Kawamoto, H., and Sankai, Y. (2005). Power assist method based on phase sequence and muscle force condition for hal. Adv. Robot. 19, 717-734. doi: 10.1163/1568553054455103

Kerrigan, D. C., Riley, P. O., Lelas, J. L., and Della Croce, U. (2001). Quantification of pelvic rotation as a determinant of gait. Arch. Phys. Med. Rehabil. 82, 217-220. doi: 10.1053/apmr.2001.18063

Kottke, F., Halpern, D., Easton, J., Ozel, A., and Burrill, C. (1978). The training of coordination. Arch. Phys. Med. Rehabil. 59, 567-572.

Kubota, S., Abe, T., Fujii, K., Marushima, A., Ueno, T., Haginoya, A., et al. (2016). Improvement of walking ability using hybrid assistive limb training in a patient with severe thoracic myelopathy caused by ossification of the posterior longitudinal ligament - a case report. J. Spine 1, 1-3. doi: 10.4172/2165-7939.S7-003

Kubota, S., Abe, T., Kadone, H., Fujii, K., Shimizu, Y., Marushima, A., et al. (2019). Walking ability following hybrid assistive limb treatment for a patient with chronic myelopathy after surgery for cervical ossification of the posterior longitudinal ligament. J. Spinal Cord Med. 42, 128-136. doi: 10.1080/10790268.2017.1313932

Kubota, S., Nakata, Y., Eguchi, K., Kawamoto, H., Kamibayashi, K., Sakane, M., et al. (2013). Feasibility of rehabilitation training with a newly developed wearable robot for patients with limited mobility. Arch. Phys. Med. Rehabil. 94, 1080-1087. doi: 10.1016/j.apmr.2012.12.020

Levangie, P. K., and Norkin, C. C. (2005). Joint Structure and Function: A Comprehensive Analysis. Philadelphia, PA: F. A. Davis Company.

Maezawa, Y., Uchida, K., and Baba, H. (2001). Gait analysis of spastic walking in patients with cervical compressive myelopathy. J. Orthopaed. Sci. 6, 378-384. doi: $10.1007 / \mathrm{s} 007760170002$

Mao, Y., Lo, W. L., Xu, G., Li, L. S., Li, L., and Huang, D. (2015). Reduced knee hyperextension after wearing a robotic knee orthosis during gait training - a case study. Biomed. Mater. Eng. 26, S381-S388. doi: 10.3233/BME-151326

Matsumiya, K. (2021). Awareness of voluntary action, rather than body ownership, improves motor control. Sci. Rep. 11, 1-14. doi: 10.1038/s41598-020-79910-x

Matsunaga, S., and Sakou, T. (2006). "OPLL: disease entity, incidence, literature search, and prognosis," in OPLL: Ossification of the Posterior Longitudinal Ligament, eds K. Yonenobu, K. Nakamura, and Y. Toyama (Tokyo: Springer), 11-17. doi: 10.1007/978-4-431-32563-5_3

Matsunaga, S., and Sakou, T. (2012). Ossification of the posterior longitudinal ligament of the cervical spine: etiology and natural history. Spine 37, 309-314. doi: 10.1097/BRS.0b013e318241ad33

McKinley, W. O., Tellis, A. A., Cifu, D. X., Johnson, M. A., Kubal, W. S., Keyser-Marcus, L., et al. (1998). Rehabilitation outcome of individuals with nontraumatic myelopathy resulting from spinal stenosis. J. Spinal Cord Med. 21, 131-136. doi: 10.1080/10790268.1998.11719521

Mizuno, J., and Nakagawa, H. (2006). Ossified posterior longitudinal ligament: management strategies and outcomes. Spine J. 6(6 Suppl.), 282-288. doi: 10.1016/j.spinee.2006.05.009

Mulroy, S., Gronley, J. A., Weiss, W., Newsam, C., and Perry, J. (2003). Use of cluster analysis for gait pattern classification of patients in the early and late recovery phases following stroke. Gait Post. 18, 114-125. doi: 10.1016/S0966-6362(02)00165-0 
Nilsson, A., Vreede, K. S., Häglund, V., Kawamoto, H., Sankai, Y., and Borg, J. (2014). Gait training early after stroke with a new exoskeleton - The hybrid assistive limb: a study of safety and feasibility. J. Neuroeng. Rehabil. 11, 1-10. doi: 10.1186/1743-0003-11-92

Oyinkan Marquis, B., and Capone, P. M. (2016). Myelopathy. Handb. Clin. Neurol. 136, 1015-1026. doi: 10.1016/B978-0-444-53486-6.00052-1

Perry, J., and Burnfield, J. (2010). Gait Analysis, Normal and Pathological Function. Thorofare, NJ: Slack Incorporated.

Perry, J., Ireland, M. L., Gronley, J., and Hoffer, M. (1986). Predictive value of manual muscle testing and gait analysis in normal ankles by dynamic electromyography. Foot Ankle 6, 254-259. doi: 10.1177/1071100786006 00506

Plautz, E. J., Milliken, G. W., and Nudo, R. J. (2000). Effects of repetitive motor training on movement representations in adult squirrel monkeys: role of use versus learning. Neurobiol. Learn. Mem. 74, 27-55. doi: 10.1006/nlme.1999.3934

Puentes, S., Kadone, H., Kubota, S., Abe, T., Shimizu, Y., Marushima, A., et al. (2018). Reshaping of gait coordination by robotic intervention in myelopathy patients after surgery. Front. Neurosci. 12:99. doi: 10.3389/fnins.2018.00099

Sakakima, H., Ijiri, K., Matsuda, F., Tominaga, H., Biwa, T., Yone, K., et al. (2013). A newly developed robot suit hybrid assistive limb facilitated walking rehabilitation after spinal surgery for thoracic ossification of the posterior longitudinal ligament: a case report. Case Rep. Orthoped. 2013, 1-4. doi: $10.1155 / 2013 / 621405$

Sankai, Y., and Sakurai, T. (2018). Exoskeletal cyborg-type robot. Sci. Robot. 3:17. doi: $10.1126 /$ scirobotics.aat3912

Sczesny-Kaiser, M., Höffken, O., Aach, M., Cruciger, O., Grasmücke, D., Meindl, R., et al. (2015). HAL®exoskeleton training improves walking parameters and normalizes cortical excitability in primary somatosensory cortex in spinal cord injury patients. J. Neuroeng. Rehabil. 12, 1-11. doi: 10.1186/s12984-015-0058-9 Seidenwurm, D. (2008). Myelopathy. Am. J. Neuroradiol. 29, 1032-1034.

Shimizu, Y., Kadone, H., Kubota, S., Suzuki, K., Abe, T., Ueno, T., et al. (2017). Voluntary ambulation by upper limb-triggered $\mathrm{HAL}^{\circledR}$ in patients with complete Quadri/paraplegia due to chronic spinal cord injury. Front. Neurosci. 11:649. doi: 10.3389/fnins.2017.00649
Shimizu, Y., Nakai, K., Kadone, H., Yamauchi, S., Kubota, S., Ueno, T., et al. (2018)

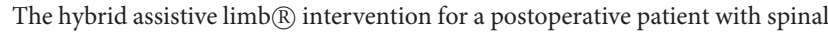
dural arteriovenous fistula and chronic spinal cord injury: a case study. J. Spinal Cord Med. 41, 710-717. doi: 10.1080/10790268.2017.1329916

Tan, C. K., Kadone, H., Watanabe, H., Marushima, A., Yamazaki, M., Sankai, Y., et al. (2018). Lateral symmetry of synergies in lower limb muscles of acute post-stroke patients after robotic intervention. Front. Neurosci. 12:276. doi: 10.3389/fnins.2018.00276

Tejima, N. (2001). Rehabilitation robotics: a review. Adv. Robot. 14, 551-564. doi: 10.1163/156855301742003

Verma, R., Arya, K. N., Sharma, P., and Garg, R. K. (2012). Understanding gait control in post-stroke: implications for management. J. Bodyw. Mov. Ther. 1, 14-21. doi: 10.1016/j.jbmt.2010.12.005

Wall, A., Wall, A., Borg, J., Borg, J., Palmcrantz, S., and Palmcrantz, S. (2015) Clinical application of the hybrid assistive limb (HAL) for gait training-A systematic review. Front. Syst. Neurosci. 9:48. doi: 10.3389/fnsys.2015.00048

Woltring, H. (1986). A Fortran package for generalized, cross-validatory spline smoothing and differentiation. Adv. Eng. Softw. 8, 104-113. doi: 10.1016/0141-1195(86)90098-7

Conflict of Interest: YSa is the C.E.O., shareholder, and director of CYBERDYNE Inc., which produces the robot suit HAL. CYBERDYNE was not involved in the study design, data collection, analysis, writing, or submission of this article.

The remaining authors declare that the research was conducted in the absence of any commercial or financial relationships that could be construed as a potential conflict of interest.

Copyright (C) 2021 Ezaki, Kadone, Kubota, Abe, Shimizu, Tan, Miura, Hada, Sankai, Koda, Suzuki and Yamazaki. This is an open-access article distributed under the terms of the Creative Commons Attribution License (CC BY). The use, distribution or reproduction in other forums is permitted, provided the original author(s) and the copyright owner(s) are credited and that the original publication in this journal is cited, in accordance with accepted academic practice. No use, distribution or reproduction is permitted which does not comply with these terms. 